\title{
REVIEW
}

\section{Sarcopenic obesity in fatty liver}

\author{
Manuela Merli, Barbara Lattanzi, and Francesca Aprile
}

\begin{abstract}
Purpose of review
Nonalcoholic fatty liver disease (NAFLD) and nonalcoholic steato hepatitis have an increasing prevalence among liver diseases. Overweight and obesity are frequently associated conditions in patients with fatty liver. Skeletal muscle mass depletion may also coexist with chronic liver disease even in obese patients. This review will focus on the relationship between sarcopenic obesity and fatty liver.
\end{abstract}

\section{Recent findings}

Obesity and sarcopenia are frequently encountered in patients with NAFLD. Adipose tissue is able to release molecules (adipokines) that regulate lipid metabolism, interact with insulin sensitivity and may contribute to induce fibrogenesis in the liver. Skeletal muscle tissue is able to secrete myokines regulating muscle metabolism and insulin sensitivity. Myokines perturbation has been reported to influence adipose tissue mass and fat deposition in the liver. Sarcopenia has been reported as independent risk factor for the development of NAFLD, and for a more severe liver fibrosis in patients with NAFLD.

\section{Summary}

The interaction between skeletal muscle, adipose tissue and the liver may play a role in the development of NAFLD. Sarcopenia and sarcopenic obesity are risk factors for the development of fatty liver and associated with more severe liver fibrosis. Management is not standardized, but dietary counseling and physical training have been proposed as promising strategies. Bariatric surgery may be considered in patients with severe 'resistant' obesity.

\section{Keywords}

insulin resistance, nonalcoholic fatty liver disease, nonalcoholic steatohepatitis, sarcopenia, sarcopenic obesity

\section{SARCOPENIC OBESITY AND NONALCOHOLIC FATTY LIVER DISEASE: THE MAGNITUDE OF THE PROBLEM}

In the last decade, nonalcoholic fatty liver disease (NAFLD) is becoming the most common cause of chronic liver disease and liver failure in Western countries [1]. The prevalence of NAFLD is estimated to range between 20 and $30 \%$ in the general population, about $30 \%$ of these patients will develop a nonalcoholic steato hepatitis (NASH) and 30-40\% of them will progress into liver fibrosis and cirrhosis [2]. A recent meta-analysis analysis of studies involving more than 8.5 million people from 22 different countries showed that more than $80 \%$ of patients with NASH are overweight or obese [3]. At the same time some studies reported a higher prevalence of sarcopenia, a condition of progressive and generalized loss of muscle mass and strength, in patients affected by NAFLD [4"',5"']. It is still under debate if these conditions, 'fat gain' and 'muscle loss', may be involved among the causes of liver fat accumulation or if they should be considered mainly a bystander. Significantly, in some of these patients skeletal muscle loss and increase in adipose tissue may even coexist, evolving to the scenario of the so called sarcopenic obesity.

The term sarcopenic obesity was firstly introduced by Baumgartner et al. in the elderly population, describing an interplay between obesity and sarcopenia related to progressive sedentarism and physical inactivity and a consequent reduction of energy expenditure. The first observation that sarcopenia, and eventually sarcopenic obesity, could be involved in NAFLD came from a large population study, the Korean Sarcopenic Obesity Study [6]. The authors examined 452 apparently healthy adults enrolled in a prospective observational cohort study. The presence of sarcopenia was associated with an increased risk of developing NAFLD. Sarcopenic obesity has been also reported in patients affected by advanced

Department of Translational and Precision Medicine, Sapienza University of Rome, Rome, Italy

Correspondence to Manuela Merli, Department of Translational and Precision Medicine, Sapienza University of Rome, Viale dell'Università 37, Rome, Italy. E-mail: Manuela.merli@uniroma1.it

Curr Opin Clin Nutr Metab Care 2019, 22:000-000

DOI:10.1097/MCO.0000000000000558 


\section{KEY POINTS}

- Prevalence of sarcopenic obesity and NAFLD is increasing and a possible interplay of the two conditions is suggested.

- A muscle-liver-adipose tissue axis is proposed as a common pathophysiological background.

- Sarcopenic obesity may accelerate liver fibrosis in patients with fatty liver.

- Treatment of sarcopenic obesity through nutritional counseling and an increase in physical activity is advisable in patients with NAFLD.

- Patients with severe obesity undergoing bariatric surgery may improve liver fibrosis.

chronic liver disease and has gained remarkable interest being associated with higher mortality in patients with liver cirrhosis $\left[7^{*}, 8,9\right]$.

\section{DEFINITION OF SARCOPENIC OBESITY}

A consensus definition for sarcopenic obesity is lacking and should derive from the knowledge of how to diagnose patients with sarcopenia and obesity. The European Working Group on Sarcopenia in Older People consider sarcopenia in presence of alteration of both muscle mass and strength, however, more commonly, sarcopenia is diagnosed taking account the muscle mass only. When a computed tomography (CT) scan, performed as for other indication, is available sarcopenia is evaluated through the skeletal muscle index (SMI) by measuring the total abdominal muscle area at L3 $[10,11]$.

CT scan is also able to inform about the proportion of intermuscular and intramuscular fat, the socalled myosteatosis [8]. Myosteatosis increases with age and adiposity and it is associated with metabolic abnormalities, decreased strength and mobility $[8,12]$. In patients with advanced liver disease myosteatosis is associated with a decreased muscle function and worse median survival [8].

If a CT scan is not available, the evaluation of the appendicular SMI (ASMI) by dual energy $\mathrm{x}$-ray absorptiometry could be a possible alternative. bioelectrical impedance analysis (BIA) and anthropometry are other complementary tools that can be utilized by trained staff. All these methods need to apply threshold values derived from a sex and age matched population. For CT-SMI threshold values were derived from inactive oncologic patients or from a population of patients with liver disease listed for liver transplantation according to their mortality [13] ASMI, BIA, and anthropometric normal values are generally available.

Obesity is defined by a BMI of at least $30 \mathrm{~kg} / \mathrm{m}^{2}$ in white populations and at least $25 \mathrm{~kg} / \mathrm{m}^{2}$ in Asian population, with further subclasses into Class I (30$\left.34.9 \mathrm{~kg} / \mathrm{m}^{2}\right)$, II $\left(35-39.9 \mathrm{~kg} / \mathrm{m}^{2}\right)$, and III $\left(\geq 40 \mathrm{~kg} / \mathrm{m}^{2}\right)$ obesity [14]. However, BMI does not give information about the relative proportions of fat and lean body mass. Considering a similar BMI, women have a higher proportion of adipose tissue while men have a higher proportion of lean muscle mass; furthermore, fat accumulation translates into increased subcutaneous adipose tissue (SAT) in women and in increased visceral adipose tissue (VAT) in men. Therefore, the evaluation of SAT and VAT would be more appropriate, when possible, to better characterize these patients. For instance, the deleterious effects of obesity, including the development of NASH, are strongly associated with an increased VAT $[15,16]$. VAT compared with SAT is richer in cells, particularly large adipocytes, which are metabolically active, as well as inflammatory and immune cells therefore, it is important to distinguish these two components. However, VAT and SAT can be evaluated only through cross-sectional CT scan.

\section{THE MUSCLE-LIVER-ADIPOSE TISSUE AXIS}

NAFLD and sarcopenic-obesity share a similar pathophysiological background. Common findings in both these conditions are: increased proinflammatory markers, decreased physical activity, reduced protein intake, disease-related reduction in testosterone and growth hormone levels [17"]. The possible interplay between adipose tissue, muscle mass, and liver, leading to sarcopenic obesity and NAFLD is shown in Fig. 1.

The pathogenesis of sarcopenic obesity in liver disease is multifaceted and further research is required to clarify both clinical and molecular aspects and to identify the mechanism of interplay among muscle, liver, and adipose tissue $\left[4^{-\cdot}, 18,19\right]$.

Until recently, adipose tissue has been mainly considered as an energy storage deposit, but in recent years this view has been largely modified. Indeed adipose tissue has been found to be able to release proteins, hormones, cytokines, and growth factors. These molecules, also known as adipokines, may act through autocrine-pathways that regulate lipid accumulation and adipocyte differentiation. In particular, adiponectin, ameliorates insulin sensitivity, and its reduction in obesity contributes to insulin resistance and glucose intolerance [20]. Moreover, being adiponectin an antisteatotic, anti-inflammatory and antifibrotic adipokine, low 


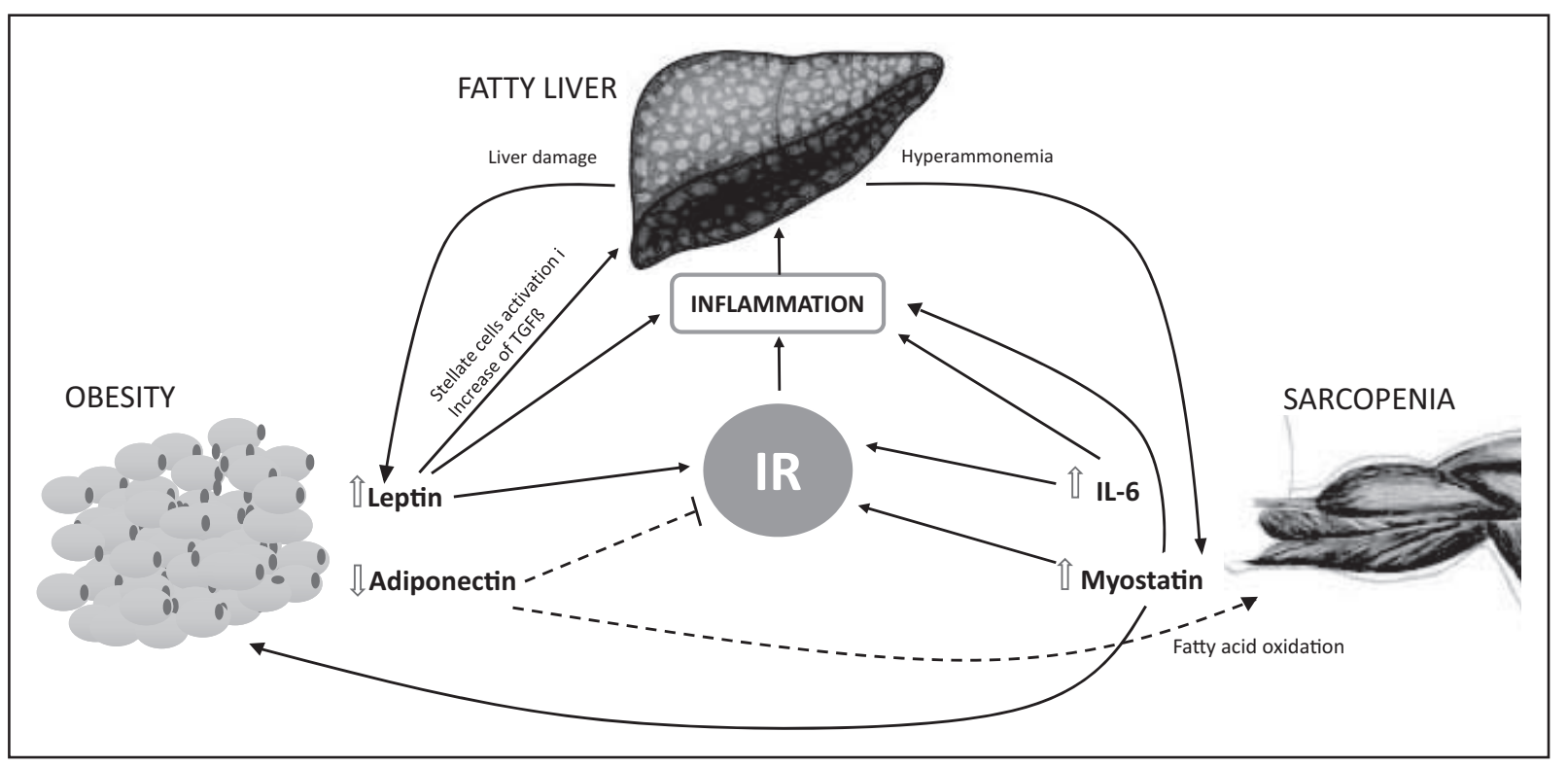

FIGURE 1. A muscle-liver-adipose tissue axis has been suggested as a possible cause of sarcopenic obesity and nonalcoholic fatty liver disease interplay. In obesity there is a reduction in adiponectin and an increase in leptin levels that contribute to reduced insulin sensitivity and glucose intolerance, increased inflammation and sarcopenia. At the same time leptin may induce the activation of hepatic stellate cells and these cells are involved in liver fibrosis. Activated hepatic stellate cells are also able of producing leptin causing the persistence of high levels. Liver disease may also contribute to sarcopenia through hyperammoniemia, high levels of myostatin induce proteostasis in skeletal muscle. Myostatin also increase adipose tissue mass and decreases adiponectin secretion. IL-6 contributes to inflammation and glucose intolerance. IR, insulin resistance; TGF $\beta$, transforming growth factor $\beta$.

adiponectin levels may predispose to fatty liver and advanced hepatic injury $[20,21]$. Leptin, being a mitogenic substance that stimulates activated hepatic stellate cells division and enhances the synthesis of inflammatory and profibrogenic factors in these cells, also contributes to fibrogenesis in chronic liver diseases. At the same time, activated hepatic stellate cells are capable of producing leptin, and this is supposed to further maintain the mechanism leading to liver fibrosis [22]. Finally, leptin also induces an increased synthesis of transforming growth factor $\beta$ (TGFß) in Kupffer cells, which contributes to fibrosis progression.

Skeletal muscle can also be seen as an endocrine organ secreting various myokines [23]. A number of animal studies have shown that myostatin, a TGFß superfamily member, which was originally discovered as a regulator of skeletal muscle mass, above the role of regulating skeletal muscle metabolism, has also significant hepatic effects. Blocking myostatin causes not only an increase in muscle mass, but also improves insulin resistance and protects animal models from the development of fatty liver $[24,25]$. It has been reported that adiponectin receptors in muscle are involved in the regulation of insulin signaling and are able to increase, at the same time, fatty acid (FA) oxidation; however the adiponectin-myostatin cross-talk has never been proven in details [26]. Since myostatin increases adipose tissue mass, and this, in turn, decreases adiponectin secretion, it can be speculated that the liver-muscle-adipose tissue perturbation may begin in the skeletal muscle and act on both liver and adipose tissue. IL-6 is another myokine that potentially regulates hepatic FA oxidation via an adenosine monophosphate-activated protein kinase dependent mechanism. Secretory and signaling perturbations of other myokines (myonectin and irisin) that regulate lipid and glucose metabolism have been suggested to contribute to the development of insulin resistance and fatty liver [27] in patients with NAFLD.

\section{THE IMPACT OF SARCOPENIC OBESITY IN NONALCOHOLIC FATTY LIVER DISEASE AND LIVER CIRRHOSIS}

In elderly people, sarcopenic obesity is associated with a worse prognosis in terms of increased risk of metabolic and cardiovascular disease, increased healthcare costs and mortality [28-30]. Obesity, via insulin resistance and systemic inflammation, is associated with a worse prognosis and higher fibrosis in patients with NASH [1,31]. However, recent studies have shown that also sarcopenia is 
related to the progression of NAFLD-related fibrosis [32"-]. Petta et al. examined 225 patients with histological diagnosis of NAFLD and documented that the prevalence of sarcopenia was independently associated with the severity of fibrosis, being severe fibrosis (F3-F4) more than doubled in sarcopenic patients ( 48.3 vs. $20.4 \%$ in fibrosis $\leq \mathrm{F} 2, P<0.001$ ). Furthermore, this study confirmed a correlation between obesity and sarcopenia [4"']. Hong et al. [6] demonstrated a higher risk of NAFLD in those with lower muscle mass after adjusting for confounding factors such as insulin resistance and inflammation. The individuals with sarcopenia presented a higher prevalence of metabolic syndrome, higher C-reactive protein levels, and higher body fat mass when compared with those without sarcopenia. Similar results were reported in another study [33]. More recently, Lee et al. [34] observed that sarcopenia was associated with significant liver fibrosis in patients with NAFLD (odds ratio 0.52$0.67 ; P<0.01$ ), independently of obesity and insulin resistance. However, it is important to highlight that in this study liver fibrosis was assessed through noninvasive scores such as Forns, fibrosis-4 index, and NAFLD fibrosis score. These data were confirmed in a recent study conducted by Koo et al. in which 309 patients were evaluated. A multivariate analysis adjusted for age, sex, BMI, hypertension, diabetes, and smoking status demonstrated that in the group of biopsy-proven NAFLD, patients with sarcopenia were more likely to develop NASH than those without sarcopenia [odds ratio, 2.28; $95 \%$ confidence interval (CI), 1.21-4.30] [6].

Sarcopenia and obesity are well known negative prognostic factor in liver cirrhosis [10,35,36]. Existing studies in cirrhosis are limited, but they have reported a quite high prevalence of sarcopenic obesity, from 5 to $35 \%$, and an association with worse prognosis and increased mortality $[8,9,37]$. A further study documented a worse prognosis in patients with sarcopenic obesity awaiting liver transplantation, the same study showed that patients with NASH have a six-fold increase risk of sarcopenic obesity [37].

\section{THE MANAGEMENT OF SARCOPENIC OBESITY}

Though novel approaches are under investigation, there is, at present, no consensus for the treatment of sarcopenic obesity in patients with liver disease. Reasons for the lack of effective therapies are incomplete understanding of the underlying mechanisms of sarcopenia in liver disease and lack of sensitive and specific biomarkers for the diagnosis. For this reasons therapies have been mainly based on deficiency of replacement rather than on mechanistic targets. The management of obesity, in patients with liver disease, is currently based on weight loss and modifications of lifestyle. The most important proposed strategies are therefore nutritional therapy, and increased physical activity. When patients are severely obese bariatric surgery is also a possible option.

\section{Nutritional therapy}

Weight loss has been reported as a keystone element for the improvement of the histological features of NASH $[38,39]$. The best therapeutic approach is based on energy restriction obtained with a low calorie $(1200-1600 \mathrm{kcal} /$ day), low fat $(<10 \%$ of saturated FA) and low carbohydrate diet ( $<50 \%$ of total kcal) [40]. Considering that weight loss following caloric restriction in overweight/obese patients results in fat mass loss (75\% of the loss) and muscle mass loss (around 25\%), very low-calorie diets are inappropriate in liver disease patients who are likely to be also sarcopenic. A 5-10\% weight loss is the target of most lifestyle interventions. To avoid the loss of muscle mass, which is certainly harmful in patients with sarcopenia and liver disease, adequate protein intake has been recommended [41]. In older individuals, a minimum protein intake of $25-30 \mathrm{~g}$ per meal has been suggested for optimal muscle protein synthesis. In cirrhotic patients, to prevent the state of 'accelerated starvation,' patients should avoid fasting for longer than 4-6 h. Evidence supports small frequent meals and the use of a late night snack containing at least $50 \mathrm{~g}$ of complex carbohydrates as well as a source of protein [42].

\section{Exercise}

To increase energy expenditure and prevent muscle mass loss, physical activity should be always implemented in patients with sarcopenic obesity. In elderly people with sarcopenic obesity a multicomponent intervention has been proposed to obtain weight reduction and increased physical activity through counseling for changing lifestyle [43].

In NAFLD an exercise-oriented meta-analysis showed improvement in steatosis, even if the level of exercise is below that recommended for the management of obesity and even in presence of minimal or no weight loss [44]. Two other meta-analyses demonstrated exercise-related improvement of liver function and steatosis $\left[45^{\mathbf{*}}, 46\right]$. Notably, one of these studies reported that exercise was more beneficial in severely obese individuals and confirmed that the effect was not associated with the intensity of the intervention (number and duration of sessions, and period of training intervention) or the alteration in diet [46]. 
In liver cirrhosis, some studies suggested a beneficial effect of exercise training associated with branched chain amino acids supplements in improving nutritional status [47"]. A small randomized pilot trial, while giving leucine supplements $10 \mathrm{~g} /$ day orally for 12 weeks in all participants, proposed exercise training in eight and no physical intervention in nine cirrhotic patients (controls). Lower thigh circumference and the 6-min walking test both improved significantly $(P=0.01)$ only in those cirrhotic patients who combined exercise training with Leucine supplements [48].

\section{Bariatric surgery}

In patients with 'resistant' obesity, bariatric surgery has proven to be effective for achieving sustained weight loss and can reverse risk factors that contribute to the pathogenesis of NAFLD, including dyslipidemia, insulin resistance, and inflammation, making it a promising treatment option for NAFLD. A recent systematic review and meta-analysis examining data from 32 cohort studies comprising 3093 biopsy specimens, found that patients' mean NAFLD activity score was reduced significantly after bariatric surgery (mean difference, 2.39; 95\% CI, 1.58-3.20; $P<0.001)$ [19].

There are limited data on the effect of bariatric surgery in patients with sarcopenic obesity. The risk of excessive loss of muscle mass exists, especially if weight loss is rapid and if bariatric surgery is not followed by a regular exercise program [49]. Since there is no available randomized controlled trial for bariatric surgery in patients with sarcopenic obesity, the best evidence to date comes from a 12-month prospective cohort study in which the effect of bariatric surgery (gastric bypass or sleeve gastrectomy) was evaluated in a cohort of morbidly obese patients comparing sarcopenic with nonsarcopenic patients [50]. Bariatric surgery resulted in similar weight loss and similar improvement in comorbidity (i.e., type 2 diabetes mellitus, NAFLD, hypertension, dyslipidemia, obstructive sleep apnea syndrome, and arthritis) in both groups. Notably, muscle mass was not different between groups 12 months after surgery, thus implying that sarcopenic patients did not lose more muscle mass despite similar weight loss.

In conclusion, sarcopenic obesity and NAFLD are strictly correlated and the interrelation between muscle, adipose tissue, and liver, results in a vicious circle in which sarcopenia, obesity, and liver disease, contribute one to the other. Sarcopenic obesity is a negative prognostic factor in patients with NAFLD therefore this condition needs to be identified and possibly treated. Dietary and lifestyle counseling are, at present, the main recommended approach. In severely obese patients bariatric surgery needs to be considered.

\section{Acknowledgements}

None.

\section{Financial support and sponsorship}

None.

\section{Conflicts of interest}

There are no conflicts of interest.

\section{REFERENCES AND RECOMIMENDED}

\section{READING}

Papers of particular interest, published within the annual period of review, have been highlighted as:

- of special interest

-1. of outstanding interest

1. Diehl AM, Day C. Cause, pathogenesis, and treatment of nonalcoholic steatohepatitis. N Engl J Med 2017; 377:2063-2072.

2. Dietrich $P$, Hellerbrand $C$. Nonalcoholic fatty liver disease, obesity and the metabolic syndrome. Best Pract Res Clin Gastroenterol 2014; 28:637-653.

3. Younossi $Z M$, Koenig $A B$, Abdelatif $D$, et al. Global epidemiology of nonalcoholic fatty liver disease - meta-analytic assessment of prevalence incidence, and outcomes. Hepatology 2016; 64:73-84.

4. Petta S, Ciminnisi S, Di Marco V, et al. Sarcopenia is associated with severe

- liver fibrosis in patients with nonalcoholic fatty liver disease. Aliment Pharmacol Ther 2017; 45:510-518.

This is an interesting study showing the independent role of sarcopenia on live damage. In spite of the higher prevalence of visceral obesity among nonalcoholic fatty liver disease (NAFLD) patients with sarcopenia, severe hepatic fibrosis was similar in obese and nonobese patients.

5. Koo BK, Kim D, Joo SK, et al. Sarcopenia is an independent risk factor for

-n nonalcoholic steatohepatitis and significant fibrosis. J Hepatol 2017 $66: 123-131$.

The study confirms, in a large-sample size, the independent association of nonalcoholic steato hepatitis and significant liver fibrosis with sarcopenia. The study therefore suggests that other mechanisms, beyond visceral obesity, may contribute to the development of liver damage. This is one of the first studies highlighting a role of sarcopenia in NAFLD patients.

6. Hong HC, Hwang SY, Choi HY, et al. Relationship between sarcopenia and nonalcoholic fatty liver disease: the Korean Sarcopenic Obesity Study. Hepatology $2014 ; 59: 1772-1778$.

7. Eslamparast T, Montano-Loza AJ, Raman M, et al. Sarcopenic obesity in - cirrhosis - the confluence of 2 prognostic titans. Liver Int 2018 38:1706-1717.

The review provides a comprehensive understanding of the impact of sarcopenic obesity in cirrhotic patients. On the contrary the available studies are still scarce. Higher rates of mortality and morbidity are however present when sarcopenia and obesity coexist in patients with liver cirrhosis.

8. Montano-Loza AJ, Angulo P, Meza-Junco J, et al. Sarcopenic obesity and myosteatosis are associated with higher mortality in patients with cirrhosis. J Cachexia Sarcopenia Muscle 2016; 7:126-135.

9. Hara N, Iwasa M, Sugimoto R, et al. Sarcopenia and sarcopenic obesity are prognostic factors for overall survival in patients with cirrhosis. Intern Med 2016; 55:863-870.

10. Montano-Loza AJ, Meza-Junco J, Prado CM, et al. Muscle wasting is associated with mortality in patients with cirrhosis. Clin Gastroenterol Hepatol 2012; 10:166-173.

11. Giusto $M$, Lattanzi $B$, Albanese $C$, et al. Sarcopenia in liver cirrhosis: the role of computed tomography scan for the assessment of muscle mass compared with dual-energy X-ray absorptiometry and anthropometry. Eur J Gastroenterol Hepatol 2015; 27:328-334.

12. Correa-de-Araujo R, Harris-Love MO, Miljkovic I, et al. The need for standardized assessment of muscle quality in skeletal muscle function deficit and other aging-related muscle dysfunctions: a symposium report. Front Physiol 2017; 15:87.

13. Carey EJ, Lai JC, Wang CW, et al., Fitness, Life Enhancement, and Exercise in Liver Transplantation Consortium. A multicenter study to define sarcopenia in patients with end-stage liver disease. Liver Transpl 2017; 23:625-633.

14. World Health Organization (WHO). Obesity and overweight factsheet from the WHO. Health 2017; 2017. 


\section{Translational research in wasting diseases}

15. Yu SJ, Kim W, Kim D, et al. Visceral obesity predicts significant fibrosis in patients with nonalcoholic fatty liver disease. Medicine (Baltimore) 2015; 94:e2159.

16. Merli M, Durand F. Muscle mass vs. adipose tissue to predict outcome in cirrhosis: which matters and in which patients? J Hepatol 2018; 69:567-569.

17. Tovo CV, Fernandes SA, Buss $C$, et al. Sarcopenia and nonalcoholic fatty liver

disease: is there a relationship? A systematic review. World J Hepatol 2017; 9:326.

The review focuses on the association between sarcopenia and NAFLD, that is independent from obesity and insulin resistance. From this starting point, new studies are needed to investigate the pathophysiology of this relationship and strategies to prevent consequences.

18. Merli M, Dasarathy S. Sarcopenia in non-alcoholic fatty liver disease: targeting the real culprit? J Hepatol 2015; 63:309-311.

19. Lee $Y$, Doumouras AG, Yu J, et al. Complete resolution of nonalcoholic fatty liver disease after bariatric surgery: a systematic review and meta-analysis. Clin Gastroenterol Hepatol 2018; 13: pii: S1542-3565(18)31138-8. doi: 10.1016/j.cgh.2018.10.017. [Epub ahead of print].

20. Buechler C, Wanninger J, Neumeier M. Adiponectin, a key adipokine in obesity related liver diseases. World J Gastroenterol 2011; 17:2801-2811.

21. Buechler $\mathrm{C}$, Haberl EM, Rein-Fischboeck $\mathrm{L}$, et al. Adipokines in liver cirrhosis. Int J Mol Sci 2017; 18:1392.

22. Duan XF, Tang $\mathrm{P}, \mathrm{Li} \mathrm{Q}$, et al. Obesity, adipokines and hepatocellular carcinoma. Int J Cancer 2013; 133:1776-1783.

23. Henningsen J, Rigbolt KT, Blagoev $B$, et al. Dynamics of the skeletal muscle secretome during myoblast differentiation. Mol Cell Proteomics 2010; 9:2482-2496

24. Bonala $\mathrm{S}$, McFarlane $\mathrm{C}$, Ang J, et al. Pid1 induces insulin resistance in both human and mouse skeletal muscle during obesity. Mol Endocrinol 2013; 27:1518-1535.

25. Zhang $C$, McFarlane $C$, Lokireddy $S$, et al. Myostatin-deficient mice exhibit reduced insulin resistance through activating the AMP-activated protein kinase signalling pathway. Diabetologia 2011; 54:1491-1501.

26. Dasarathy $S$. Is the adiponectin-AMPK-mitochondrial axis involved in progression of nonalcoholic fatty liver disease? Hepatology 2014; 60:22-25.

27. Polyzos SA, Kountouras J, Anastasilakis $A D$, et al. Irisin in patients with nonalcoholic fatty liver disease. Metabolism 2014; 63:207-217.

28. Park SH, Park JH, Song PS, et al. Sarcopenic obesity as an independent risk factor of hypertension. J Am Soc Hypertens 2013; 7:420-425.

29. Kim TN, Choi KM. The implications of sarcopenia and sarcopenic obesity on cardiometabolic disease. J Cell Biochem 2015; 116:1171-1178.

30. Tian S, Xu Y. Association of sarcopenic obesity with the risk of all-cause mortality: a meta-analysis of prospective cohort studies. Geriatr Gerontol Int 2016; 16:155-166.

31. Berentzen TL, Gamborg M, Holst $C$, et al. Body mass index in childhood and adult risk of primary liver cancer. J Hepatol 2014; 60:325-330.

32. Pan $X$, Han $Y$, Zou $T$, et al. Sarcopenia contributes to the progression of nonalcoholic fatty liver disease-related fibrosis: a meta-analysis. Dig Dis 2018; $36: 427-436$

The work adds some new results to the established relationship between sarcopenia and NAFLD. This analysis demonstrated the important role of sarcopenia for the onset of NAFLD and for the progression of NAFLD-related fibrosis.

33. Lee $\mathrm{YH}$, Jung KS, Kim SU, et al. Sarcopaenia is associated with NAFLD independently of obesity and insulin resistance: nationwide surveys (KNHANES 2008-2011). J Hepatol 2015; 63:486-493.
34. Lee $\mathrm{YH}$, Kim SU, Song $\mathrm{K}$, et al. Sarcopenia is associated with significant liver fibrosis independently of obesity and insulin resistance in nonalcoholic fatty liver disease: nationwide surveys (KNHANES 2008-2011). Hepatology 2016; 63:776-786.

35. Berzigotti A, Abraldes JG. Impact of obesity and insulin-resistance on cirrhosis and portal hypertension. Gastroenterol Hepatol 2013; 36:527-533.

36. Berzigotti A, Garcia-Tsao G, Bosch J, et al., Portal Hypertension Collaborative Group. Obesity is an independent risk factor for clinical decompensation in patients with cirrhosis. Hepatology 2011; 54:555-561.

37. Carias S, Castellanos AL, Vilchez V, et al. Nonalcoholic steatohepatitis is strongly associated with sarcopenic obesity in patients with cirrhosis undergoing liver transplant evaluation. J Gastroenterol Hepatol 2016; $31: 628-633$.

38. Haufe $S$, Engeli $S$, Kast $P$, et al. Randomized comparison of reduced fat and reduced carbohydrate hypocaloric diets on intrahepatic fat in overweight and obese human subjects. Hepatology 2011; 53:1504-1514.

39. Asrih M, Jornayvaz FR. Diets and nonalcoholic fatty liver disease: the good and the bad. Clin Nutr 2014; 33:186-190.

40. Italian Association for the Study of the Liver (AISF). AISF position paper on nonalcoholic fatty liver disease (NAFLD): updates and future directions. Dig Liver Dis 2017; 49:471-483.

41. The European Association for the Study of the Liver. EASL Clinical Practice Guidelines on nutrition in chronic liver disease European Association for the Study of the Liver. J Hepatol 2019; 70:172-193.

42. Tsien CD, McCullough AJ, Dasarathy $\mathrm{S}$. Late evening snack: exploiting a period of anabolic opportunity in cirrhosis. J Gastroenterol Hepatol 2012 27:430-441.

43. Bouchonville MF, Villareal DT. Sarcopenic obesity: how do we treat it? Curr Opin Endocrinol Diabetes Obes 2013; 20:412-419.

44. Keating SE, Hackett DA, George J, et al. Exercise and nonalcoholic fatty liver disease: a systematic review and meta-analysis. J Hepatol 2012, 57:157-166.

45. Katsagoni CN, Georgoulis M, Papatheodoridis GV, et al. Effects of lifestyle

- interventions on clinical characteristics of patients with nonalcoholic fatty liver disease: a meta-analysis. Metabolism 2017; 68:119-132.

The meta-analysis provides evidence that patients with NAFLD improve their liver status by exercise alone or in combination with a dietary intervention.

46. Orci LA, Gariani K, Oldani G, et al. Exercise-based interventions for nonalcoholic fatty liver disease: a meta-analysis and meta-regression. Clin Gastroenterol Hepatol 2016; 14:1398-1411.

47. Hiraoka A, Michitaka K, Kiguchi D, et al. Efficacy of branched-chain

amino acid supplementation and walking exercise for preventing sarcopenia in patients with liver cirrhosis. Eur J Gastroenterol Hepatol 2017; 29 : 1416-1423.

Despite the small number of patients and short-term evaluation, this study shows two possible strategies to prevent patients from the negative consequences of sarcopenia in cirrhosis. An interesting study that could inspire innovative clinical trial on the management of sarcopenic obesity in NAFLD patients.

48. Román E, Torrades MT, Nadal MJ, et al. Randomized pilot study: effects of an exercise programme and leucine supplementation in patients with cirrhosis. Dig Dis Sci 2014; 59:1966-1975.

49. Mathus-Vliegen EM. Prevalence, pathophysiology, health consequences and treatment options of obesity in the elderly: a guideline. Obes Facts 2012; $5: 460-483$

50. Mastino $D$, Robert $M$, Betry $C$, et al. Bariatric surgery outcomes in sarcopenic obesity. Obes Surg 2016; 26:2355-2362. 